\title{
Vorwort: Produkthaftung, Produkthaftpflichtversicherung, Betriebsorganisation und der Zweck dieser Entscheidungssammlung
}

1. Die deutsche Rechtsprechung hat fast alle für die betriebliche Praxis wichtigen Fragen der Produkthaftung bereits behandelt und geklärt. Die Entscheidungen sind aber teils überhaupt nicht bzw. nur auszugsweise veröffentlicht, teils über eine Vielzahl von juristischen Fachzeitschriften, o. ä. verstreut und ad hoc aus Anlaß eines konkreten Einzelfalls für den Nichtspezialisten nicht bzw. nur schwer erreichbar. Diese Entscheidungssammlung soll deshalb zunächst einmal dem Juristen einen schnellen Zugang zu den vorliegenden Entscheidungen ermöglichen.

2. Weiterhin ist die Entscheidungssammlung aber auch für die betriebliche Praxis im kaufmännischen und im technischen Bereich gedacht. Wegen der ständig zunehmenden Bedeutung des Produktrisikos für die Risikoexponierung der Unternehmen und darüber hinaus auch der Mitarbeiter ${ }^{1}$ wird es immer wichtiger, daß sich die potentiell Betroffenen intensiv mit diesem Fragenbereich auseinandersetzen.

a) Bei dem Thema Haftung wird sofort an die Haftpflichtversicherung gedacht. Die Produkthaftpflichtversicherung bietet zweifellos einen sehr weitgehenden Schutz. Sie muß aber immer gewisse Grenzen haben. Aus der unternehmerischen Sicht kann die Produkthaftpflichtversicherung deshalb nur ein sekundäres Instrument zur Bewältigung des Produktrisikos sein. ${ }^{2}$

aa) Die Frage nach den Grenzen der Produkthaftpflichtversicherung betrifft zunächst den nicht versicherbaren Bereich. Wenn im Fall eines Großserienschadens der volle Schadenaufwand versichert war und dadurch außer der zu Beginn des Versicherungsjahres bezahlten Versicherungsprämie keine Kostenbelastung des Herstellers erfolgte, kann es sein, daß die Verbraucher das Vertrauen in die Marke verloren haben und auf Wettbewerbsprodukte umsteigen. Durch diese Ausstrahlung des hinsichtlich des konkreten Schadenauf wands versicherungsmäßig voll abgedeckten Schadens kann sich für den Hersteller eine katastrophale Situation ergeben. Gegen derartige Ausstrahlungsrisiken kann es aber keinen Versicherungsschutz geben.

ab) Weiterhin sind die Deckungssummen- und Deckungsumfang-Grenzen der Produkthaftpflichtversicherung zu berücksichtigen. Die in Deutschland übliche, das Produktrisiko umfassende Betriebshaftpflichtversicherung stellt zwar eine sehr umfassende Deckung dar. Sie enthält aber notwendigerweise gewisse Deckungsbegrenzungund Deckungsausschlußklauseln, so daß diese Grenzen des versicherten Risikos zu beachten sind.

1 Siehe insbesondere das Spannkupplungen-Urteil (I. 86) und allgemein das Stichwort „,Mitarbeiter-Eigenhaftung "

2 Vgl. dazu Schmidt-Salzer, BB 1972/1430ff. 
Weiterhin sind die Deckungssummen und die Jahreshöchstleistungen begrenzt. Die Höhe der Versicherungsprämie wird $u$. a. durch die Höhe der pro Schadenereignis festgelegten Deckungssumme und weiterhin die pro Versicherungsjahr vom Haftpflichtversicherer zu erbringende Maximalleistung bestimmt. Deshalb erfolgt die Festlegung der Deckungssummen und der Jahreshöchstleistung im allgemeinen auf der Basis einer kaufmännisch vernünftigen Einschätzung der für das einzelne Unternehmen bestehenden Risikoexponierung. Dabei werden u. a. die Größenordnung des Unternehmens, die Höhe seines Jahresumsatzes, die Einschätzung der mit seinem Herstellungs- bzw. Vertriebskonzept verbundenen Haftungsrisiken, die Schadenerfahrungen der Vergangenheit sowie die zahlenmäßige Größenordnung der zur Placierung des konkreten Versicherungskonzeptes benötigten Prämie berücksichtigt. Bei unglücklichem Zusammentreffen mehrerer Faktoren und erst recht bei unglücklichem Zusammentreffen mehrerer Großschäden in einem Versicherungsjahr kann sich aber ergeben, daß auch an sich recht hoch angesetzte Deckungssummen bzw. Jahreshöchstleistungen im Einzelfall überschritten werden.

Hier besteht ein wichtiger Unterschied zwischen der Industrie-Feuerversicherung und der Industrie-Haftpflichtversicherung: im theoretischen Konzept der Industrie-Feuerversicherung ist es möglich, den maximalen Höchstschaden im vorhinein relativ präzise $\mathrm{zu}$ ermitteln und dementsprechend die Versicherungssummen festzusetzen. In der Industrie-Haftpflichtversicherung dagegen ist es letztlich nur eine Frage der Phantasie, welcher Schadenaufwand sich bei unglücklichem Zusammentreffen verschiedener Faktoren ergibt. Z. B. ist die gemäß § 94 ArzneimittelG vorgeschriebene Deckungssumme für die Personenschaden-Haftpflichtversicherung von in Deutschland an den Verbraucher abgegebenen Arzneimitteln mit 200 Mio DM sehr hoch angesetzt. Abgesehen von einigen wenigen Großunternehmen würden normalerweise auch große und erst recht mittelständische oder gar kleinere Unternehmen eine derartige Dekkungssumme nicht beschaffen. Der Contergan-Fall mit einem Schadenaufwand von etwa 2 Milliarden DM hat aber gezeigt, daß im Fall eines Großserien-Schadens auch eine derartige Deckungssumme unzulänglich sein kann.

Die Industrie-Haftpflichtversicherung bietet also zwar für normale und auch für außergewöhnliche Fälle eine sehr wichtige Risikoabschirmung. In Extremfällen können aber auch die Grenzen einer kaufmännisch vorsichtigen Versicherungsabdeckung überschritten sein.

b) In den letzten 10 Jahren hat das Produktrisiko völlig neue Dimensionen gewonnen. Diese Entwicklung wird mit Sicherheit weitergehen. Dies ist nicht nur eine Frage von Weiterentwicklungen des Haftungs- und des Prozeßrechts. Der wesentliche Grund liegt m. E. in der ständig zunehmenden Anspruchskenntnis der Geschädigten und im ständig zunehmenden Anspruchsbewußtsein und Anspruchsdenken. Je größer aber das Produktrisiko wird, um so höher wird der Schadenaufwand. Um so mehr steigen die von den Haftpflichtversicherern zur Versicherung des Produktrisikos benötigten Prämien. 
Um so wichtiger wird es aus der unternehmerischen Sicht, sich hinsichtlich der Bewältigung des Produktrisikos nicht nur mit der Produkthaftpflichtversicherung zu begnügen, sondern die Versicherungsdeckung zu ergänzen durch unternehmensinterne Schadenverhütungs- bzw. Schadenminderungsmaßnahmen.

Dafür wiederum ist eine möglichst genaue Kenntnis und Analyse der Produkthaftung und ihrer Ausstrahlungen auf die konkrete Risikoexponierung des einzelnen Unternehmens erforderlich. Diese Arbeit kann nicht von den Juristen geleistet werden. Vielmehr müssen die Kaufleute und die Techniker in ihren Arbeitsbereichen ein ,Parallelwissen in der Laiensphäre" über die relevanten Ausstrahlungen der Produkthaftung aufweisen. Nur dies schafft die Voraussetzung dafür, daß in der betrieblichen Praxis versucht werden kann, im Rahmen des wirtschaftlich Möglichen zugleich auch Schadenverhütung im Hinblick auf die Begrenzung der Haftungsexponierung zu betreiben. Es handelt sich hier also um wesentlich mehr als um nur betriebswirtschaftliche Fragen der Kosten/Nutzen-Bewertung z. B. von Qualitätssteuerungsmaßnahmen.

Das Ziel dieser Entscheidungssammlung und der Urteilsanmerkungen ist es, auch diesem Personenkreis den Einstieg in die Problematik zu ermöglichen. Ich habe versucht, in den Urteilsanmerkungen bei allem Streben nach einer höchstmöglichen juristischen Präzision zugleich auch eine Verständlichkeit für den Nichtjuristen zu erreichen und weiterhin im Rahmen des vom Umfang dieses Buches her Möglichen auch auf die Konsequenzen für die betriebliche Praxis hinzuweisen.

Ein kardinales Darstellungsproblem ergibt sich dabei aus den unterschiedlichen Begriffen und Sprachgebräuchen des juristischen, des technischen und des kaufmännischbetriebswirtschaftlichen Bereichs, die jeweils eigenständig ohne Rücksicht auf die Entwicklung in den Paralleldisziplinen gewachsen sind. Soweit wie möglich wurde im Rahmen der sachlichen Analyse auch eine terminologische Vereinheitlichung und im Stichwortverzeichnis eine Berücksichtigung auch der außerjuristischen Begriffswelt angestrebt.

3. Der 1975 erschienene Band I umfaßte im wesentlichen Entscheidungen, die bis Ende 1974 ergangen bzw. veröffentlicht waren, sowie zwei nachgeschobene, sehr wichtige Entscheidungen des Bundesgerichtshofs. In der Zwischenzeit sind mir weitere Entscheidungen aus diesem Zeitraum bekannt geworden, die ich wegen der Bedeutung in den Band II hereingenommen habe.

Viele Hinweise auf Entscheidungen vor allem der Instanzgerichte verdanke ich Richtern, Anwälten und Technikern. Jedem Benutzer der Entscheidungssammlung bin ich im Interesse einer möglichst umfassenden Materialerfassung für weitere Hinweise dankbar.

4. Meinem Kollegen Dr.-Ing. Egon Sattler, mit dem mich eine mehrjährige intensive Zusammenarbeit im Bereich der Schadenverhütung verbindet, danke ich auch an die- 
ser Stelle für den konstruktiv-kritischen Dialog über die Urteilsanmerkungen und viele Fragen in den Grenzbereichen zwischen Juristerei und Technik. Die Produkthaftung hat eine sozialpolitische und wirtschaftliche Bedeutung gewonnen, die sie weit über den Bereich der Juristen hinaushebt. Die mit der Produkthaftung verbundenen Fragen werden immer mehr zu einem Feld, in dem die interdisziplinäre Zusammenarbeit zwischen Juristen, Betriebswirten, Kaufleuten und Technikern sowie allen mit Fragen der Produktgestaltung beschäftigten Wissenschaftlern und Praktikern weiterer Bereiche brennend erforderlich wird. Möge diese Entscheidungssammlung in Verbindung mit den Urteilsanmerkungen dafür einen Beitrag und hoffentlich auch eine Grundlage bieten.

3300 Braunschweig

Dr. Joachim Schmidt-Salzer

Margaretenhöhe 15

im Juli 1979 